\title{
Sur les groupes de difféomorphismes des variétés différentiables.
}

Memoria di Jacqueline Lelong-Ferrand (a Parigi, Francia)

À M. Enrico Bompiani pour son Jubilé scientifique.

Itêsumé. - Nous établissons ici deux conditions. nécessaires et suffisantes, pour qu'une algèbre de LIE de champs de vecteurs tangents à une variété différentiable $V$, engendre un groupe global de diffeomorphismes de $\mathrm{V}$.

§ 1. - Une étude antérieure [1 et 2] nous permet d'énoncer le résultat suivant $\left({ }^{2}\right)$.

Proposition 1. - Pour qu' un champ de vecteurs $\xi(x)$, de classe $C_{1}^{0}$ sur une vartété $V$ de classe $C_{1}^{4}$, engendre un groupe global $\dot{a}$ un paramètre de difféomorphismes de $V$

a) il suffit $q u^{\prime}$ il existe une foniction $r(x) \geq 0$ de classe $C_{1}^{0}$ sur $V$, dont les «nombres dérivés» de Lie soient bornés et telle que l'ensemble $E_{n}$ défini par $r(x) \leq k$ soit compact queque soit $k$

b) il faut que $V$ soit la réunion d'une fanille de domaines invariants satisfaisant à la condition précédente.

Le présent article a pour but d'étendre ce résultat aux groupes de LIE de dimension finie quelconque. En fait, nous établirons deux critères différents, généralisant tous deus la proposition 1 et découlant d'un même principe général (théorème I). Dans le premier critère (théorème II) la fonction $r$ est remplacee par une application propre de $V$ dans le groupe de LIE $G$ présumé agir sur $V$, ou, plus généralement, par une application propre de $V$ dans une variété $\bar{V}$ sur laquelle $G$ agit globalement; ce critère a été déjà énoncé partiellement dans [3]. Le deuxième critère (théorème III) généralise plus étroitement la proposition 1.

(1) Dans les articles eités nous avions supposé la variéte $V$ pourvue d'une structure uniforme de variété complète. car nous avions en vue l'étude des variétés à bord. En fait ceite hypothèse est inutile et la démonstration s'applique sans modification aux variétés ouvertes (sans bord) indépendamment de la donnée de toute structure uniforme. 


\section{§ 2. Position du problème.}

Dans tout ce qui suit, nous désignerons par $l$ une variété différentiable $\left(^{2}\right)$ séparée, de classe $C^{2}$. de dimension $n$ (variétí onverte) et par $G$ nn groupe de LIE connexe de dimension $r$. Un $G$-groupe global de diffémorphismas de $V$ est defini par une application $\varphi$ de classe $C^{1} d e G \times V^{\prime}$ dans $I$ satisfai. sant a

$$
\varphi(e . x)=x
$$

et à

$$
\varphi(h g, x)=\varphi[h . \varphi(g, x)]
$$

quel que soient $x \in V$ et $g, h \in G$.

Si nous posons $\varphi^{x}(g)=\varphi(g, x)=y$ et $\psi^{g}(h)=h g$, la relation $(2,2)$ équivaut a $\varphi^{m}$ o $\psi^{g}=\varphi^{\nu}$ et entraine

$$
\delta \xi^{x}\left(L_{g}\right)=\delta \varphi^{\nu}\left(L_{e}\right)
$$

où $L_{e}$ désigne un vecteur quelconque tangent en $e$ à $G$ et $L_{\sigma}$ le champ de vecteurs invariant à droite sur $G$, défini par $L_{g}=\hat{c} \cdot q^{g}\left(L_{e}\right)$.

Nous identifierons l'algèbre de LiE $\mathfrak{g}$ de $G$ à l'algèbre de LiE des champs de vecteurs $L_{g}$ invariants à droite sur $G$; à chaque $L \in \mathfrak{g}$ correspond ainsi sur $V$ un champ de vecteurs $\xi_{L}$ defini par

$$
\xi_{L}(x)=\delta p^{x}\left(L_{e}\right)
$$

Nous supposerons désormais que pour chaque $x \in V^{\prime}$ la différentielle ó $\varphi^{x}$ est de classe $C^{1}$; alors les champs de vecteurs $\xi_{L}$ sont eux-memes de classe $C^{2}$ et l'application $\theta: L-\xi_{L}$ definit un homomorphisme de $\mathfrak{g}$ dans une algèbre de LIE de champs de vecteurs de classe $C^{1}$ sur $V$.

Inversement supposons donné un homomorphisme $\theta$ de l'algèbre de LiE de $G$ dans une algèbre de Lie de champs de vecteurs de classe $C^{1}$ sur $V$ : nous dirons $q u \theta A$ définit un $G$-groupe de transformations infinitésimales de $V$.

Le problème posé consiste à chercher les conditions que doivent remplir les données $(G$ et $\theta$ ) pour qu'il existe une application $\varphi$ de $G \times V$ dans $V$, satisfaisant à $(2,1)$ et à

$$
\delta \varphi^{x}\left(L_{\rho}\right)=\theta(L)_{\xi(g, x)} .
$$

(2) Dans [1] et [2] la variété $V$ avait été supposée seulement de classe $C_{1}^{1}$ [ce qui signifie que les changements de coordonnés, sont définis par des fonctions pourvues de dérivées du premier ordre lipschitziennes], ot les champs de vecteurs considérés, de classe $C_{1}$ [ [e'est à dire lipschitziens]. Nous avons été amenés à faire iei des bypothèses plus rentrictives. 
Si nne telle application existe, nous dirons que $A$ engendre un $G$-groupe global de difféomorphismes de $V$ : on sait en effet que les relations $(2,1)$ et $(2,3)$ entrainent $(2,2)$.

Or il est connu $\left({ }^{3}\right)$ que tout $G$-groupe de transformations infinitésimales de $V$ engendre un groupe local de diffémorphismes de $V$ : autrement dit, il existe un voisinage $\Omega$ de $\{e\} \times V$ dans $G \times V$ et une application $\varphi$ de $Q$ dans $V$, définie de manière unique, satisfaisant aux relations $(2,1)$ et $(2,3)$. Pour que le groupe local $;$ se prolonge $\left.{ }^{4}\right)$ en un groupe global, il faut et il suffit que pour chaque $a \in V$ l'application $\varphi^{a}: g \rightarrow f(g, a)$ soit prolongeable a $G$ tout entier sans cesser de satisfaire aux relations (2,3); et si $G$ est connexe, ce problème se décompose à son tour en denx tracé dans $C$.

$\left.P_{1}\right)$ Prolongement de $\varphi^{a}(g)$ le long d'un arc quelconque issu de $e$ et

$P_{2}$ ) Uniformité du prolongement ainsi défini.

Nous nous hornerons ici à étudier le problème $P_{1}$; la question $P_{2}$ est en effet résolue automatiqnement si $G$ est simplement connexe, car la valeur de $p^{a}(g)$ obtenue par prolongement le long $d^{\prime}$ un arc $\gamma$ jcignant $e$ à $g$ ne dépend que de la classe d'homotopie de $\gamma$. Or la définition du groupe infinitésimal $A$ n' fait intervenir que l'algèbre de LiE $g$ et non le groupe $G$ lui-mème: nous pouvons donc toujours supposer que $G$ est simplement con. nexe. Cependant, ponr plus de clarté, le probleme $P_{1}$ sera étudié sans cette hypothèse.

Définition. - Nous dirons que la distribution $\theta$ est régulière si quel que soit $a \in \mathrm{V}^{\gamma}$, l'application $\varphi^{a}$ est prolongeable le long de tout are issu de $e$ et trace dans $G$.

Il est possible d'étudier directement le problème $P_{1}$ et de montrer ensuite que l'application obtenue par prolongement de $\varphi^{a}$ répond aux conditions voulues quand elle est uniforme. Nous avons préféré nous ramener à un autre problème, dont l'équivalence avec $P_{1}$ (théorème 1) sera établie à l'aide des résultats de R. S. PalaIs [4]: le point de vue topologique ainsi adopté permet en effet certaines généralisations.

Notation. - Nous adoptons les principales notatiuns utilisées par R. S. PALAIS et nous désignons par $\theta^{*}$ la distribution involntive définie sur $G \times V \operatorname{par} \Theta_{(g, x)}^{*}(L)=\left\{L_{g}, \Theta(L)_{x}\right\} ;$ par $\Sigma_{a}$ la feuille intégrale de la distribu.

( ${ }^{3}$ Ce résultat, sous sa forme locale, constitue le second theorème fondamental de Lre. L'énoncé que nous donnon ici est établi par R. S. Palars dans [4], p. 36 a 58.

(4) Le groupe local $\bar{\varphi}$ est dit prolonger $\varphi$ si le domaine $Q$ de $\varphi$ contient $Q$, et si on a $\bar{\varphi}=\varphi \operatorname{sur} \underline{\mathbf{Q}}$. 
tion $\Theta^{*}$ passant par le point $\left(e, a\right.$; et par $\pi_{G}\left[\right.$ resp. $\left.\pi_{V}\right]$ la projection canonique de $G \times V$ sur $G$ [resp. sur $V$ ]. L'existence du groupe local $\varphi$ résulte alors essentiellement de la règle suivante ([4], Th. VII, p. 43):

"Pour ehaque $a \in V$ le graphe de $\varphi^{a}$ dans $G \times V$ est une sous variété ouverte et connexe de $\Sigma_{a}$, sur laquelle $\pi_{G}$ se réduit à un difféomorphisme».

On en déduit immédiatement la propriété suivante

Proposrmon 2. - Pour que la distribution $\Theta$ soit régulière, il faut et il suffit que pour chaque $a \in V$, le couple $\left(\mathbf{\triangle}_{a}, \pi_{G}\right)$ définisse un recouvrement de $G$.

La notion de distribution régulière corncide dono avec celle de distribu. tion uniforme introduite par R. S. Palais (Cf. [4], th. XVII, p. 80): la distribution $\Theta$ est régulière si, et seulement si, il existe un voisinage $U$ de $e$ dans $G$, et une application $\varphi$ de $U \times V$ dans $V$, satisfaisant à $(2,1)$ et à $(2,3)$.

\section{\$ 3. Etade topologique préliminaire.}

Nous allous d'abord montre comment la donnée d'un groupe de transformations infinitésimales détermine sur $V$ une structare uniforme $\mathscr{Q}$ et étudier les propriétés de cette structure. Nous verrons alors que la condition « $\Theta$ est régulière " équivaut à " $V$ est complète pour la structure $\mathcal{Q} \ell$ ».

Bien $q u e$ la structure $\mathscr{L}$ puisse être définie indépendamment de toute donnée métrique, il nous sera commode pour la suite de définir sur $G$ une métriqne riemannienne invariante à droite; la structure $\mathcal{Q}$ peut alors être definie par un écart $e x, y$.

Dénintron. - Deux points $x, y$ de $V$ seront dits G-équivalents s'il existe un arc $\Gamma$ tracé dans une feuille intégrale de $\Theta^{*}$ et dont la projection sur $V$ joigne $x$ à $y$.

La relation d'équivalence ainsi définie est évidemment symétrique et reflexive; il nous faut montrer qu'elle est transitive: à cet effet, nous remarquerons d'abord que s'il existe un arc $\Gamma$ tracé dans une feuille $\Sigma$ et répondant aux conditions voulues, l'are $\Gamma_{h}$ déduit de $\Gamma$ par la translation droite $R_{h}^{*}:(g, x) \rightarrow(g h, x)$ répond encore à ces conditions. Nous pouvons donc, sans restreindre la définition, imposer à $\Gamma$ d'être issu du point $(e, x)$, par conséquent, d'être contenu dans $\Sigma_{x}$.

Si le point $z$ est aussi $G$-équivalent à $x$, il existe de mème un arc $\Gamma^{\prime}$ issu de $(e, x)$ et contenu dans $\Sigma_{x}$, tel que $\pi_{V}\left(\Gamma^{\prime}\right)$ joigne $x$ à $z$; et en utilisant l'arc $\Gamma^{\prime \prime}$ constitué par la réunion de $\Gamma$ et de $\Gamma^{\prime}$ parcourus dans des sens convenables, on voit que $y$ est G-équivalent à $z$. C. q. f. d.

REMARQUE. - Nons pouvons sans modifier la relation d'equivalence précédente, imposer à $\Gamma$ d'etre de classe $C^{2} ; \pi_{G}(\Gamma)$ est alors de classe $C^{1}$. 


\footnotetext{
Definition. - Si $x$ et $y$ sont deux points G-équivalente de $\mathrm{I}^{r}$, nous poserons

$$
e(x, y)=\inf [\text { longueur } \pi G(\Gamma)]
$$
}

où $\Gamma$ désigne un arc quelconque de classe $C^{1}$ tracé dans nue feuille intégrale de $\theta^{*}$ et tel que $\pi v(\Gamma)$ joigne $x$ à $y$ (l'existence de tels arcs résultant de la remarque précédente).

Si $x$ et $y$ ne sont pas $G$-équivalents, nons poserons $e(x, y)=+\infty$.

Par le mème procédé qui nous a servi à établir la transitivité de la $G$-équivalence, on établirait sans peine que $e(x, y)$ satisfait à l'inégalité triangulaire [la translation droite $R_{h}^{*}$ ne modifie pas en effet la longueur de $\left.\pi_{(\rightarrow}[\Gamma)\right]$. Nous désignerons par $\mathcal{Q} \ell$ la structure uniforme definie sur $V$ par l'écart $e(x, y)$ : et pour éviter les confusions, la structure topologique qui se déduit de la structure de variété de $V$ sera désignée par $\mathrm{f}$.

La distance géodésique de deux points $g, h$ de $G$ (c'est à dire la borne inférieur des longueurs des ares joignant $g$ à $h$ dans b) sera désignée par $d(g, h)$.

Propriétís de LA struCture $\mathcal{Q}$.

Proposition 3. - La structure topologique associée à $\mathscr{Q}$ est plus fine $q$ ue $\mathrm{f}$.

Soit, en effet, $w$ un t-voisinage donné d'un point $a$ de $V$. La restriction de $\pi_{V} \grave{a} \Sigma_{a}$ étant $\mathfrak{f}$-continue, $S=\pi_{V}^{-1}(w) \cap \Sigma_{a}$ est un voisinage de $(e, a)$ dans $\Sigma_{a}$; et puisque la restriction de $\pi_{G}$ à $\Sigma_{a}$ est un difféomorphisme local, $\pi_{G}(S)$ est un voisinage de $e$. Il existe donc un nombre $x>0$ tel que $\pi_{G}(S)$ contienne la boule de $G$ définie par $d(e, g)<\alpha$; il en résulte que $w$ contient la boule de $V$ définie par $e(a, x)<\alpha$ : done $w$ est un $Q \mathcal{Q}$-voisinage de $a$. C. q. f. $d$.

Propositron 4. - Quel que soit le point a de $V, e(a, x)$ est une fonction semi-continue supérieurement de $x$ pour la topologie $\mathfrak{f}$.

Chaque point de $V$ admettant une base dénombralle de f-voisinages, il nous suffit d'établir que si $b \in V$ est limite (pour la topologie $f$ ) d' une suite $x_{n}$ satisfaisant à $e\left(a, x_{n}\right) \leq \alpha$, aiors $e(a, b) \leq \alpha$, quel que soit $a \in V$.

La propriété n'a besoin d'ètre dẻmontrée que si $\alpha<\infty$. Alors, les points $x$, sont $G$-équivalents a $a$, et, quel que soit $\varepsilon>0$ donné il existe dans $\aleph_{a}$ une suite d'arcs $\Gamma_{n}$. tels que $\pi_{V}\left(\Gamma_{n}\right)$ joigne $a$ à $x_{n}$ et que la longueur de $\pi_{G}\left(\Gamma_{n}\right)$ soit inférieure à $\alpha+\varepsilon$. Désignons par $g_{n}$ l'extrèmité de $\pi_{G}\left(\Gamma_{n}\right)$. La boule de $G$ définie par $d(e, g) \leq \alpha+\varepsilon$ etant compacte (') nons pouvons, par

(5) Pour une démonstration de ce fait, voir par exemple, L. C̈̈rding, . Bull. Soc. Math. France , t. 88 (1960): p. 74 . 
extraction d'une suite partielle, supposer que la suite $g_{n}$ converge vers un point $g$ de $G$, et que la série $\mathbb{S} d\left(g_{n}, g_{n+1}\right)$ est convergente. On sait (i) qu'il existe un voisinage $U$ de $e$ dans $G$ et un roisinage $w$ de $b$ dans $V$ tels que, pour chaque $x \in w$, la restriction de $\pi_{G}$ a la composante connexe de $(e, x)$ dans $\Sigma_{x} \cap \pi_{G}^{-1}(U)$ soit un difféomorphisme. Il existe d'autre part un entier $N$ tel que, pour $n \geq N$, le point $g_{n+1} g_{n}^{-1}$ puisse etre joint à $e$ par un are de longueur inférienre à $2 d\left(g_{n}, g_{n+1}\right)$ et tracé dans $U$. Par relèvement dans $\Sigma_{x_{n}}$ et translation droite, on en déduit, pour $n \geq N$, l'existence d'un arc $\mathrm{I}^{\prime}{ }_{n}$ joignant $\left(g_{n}, x_{n}\right)$ à $\left(g_{n+1}, x_{n+1}\right)$ dans $\Sigma_{a}$, et tel que la longueur de $\pi_{G}\left(\Gamma_{n}^{\prime}\right)$ soit inférieure à $2 d\left(g_{n}, g_{n+1}\right)$. La réunion de $\Gamma_{k}(k \geq N)$ et des arcs $\Gamma_{n}(n \geq k)$ constitue un arc $C_{k}$ joignant $(e, a)$ a $(g, b)$ dans $\Sigma_{a}$, et tel que la longueur de $\pi_{G}\left(C_{k}\right)$ soit inférieure à $\alpha+\varepsilon+2 \underset{n}{\Sigma} d\left(g_{n}, g_{n+1}\right)$. En faisant tendre $k$ vers l'infini, on en déduit l'inégalité $\left.e(a, b)^{n}\right)^{k} \leq \alpha+\varepsilon$, qnel que soit $\varepsilon>0$. C. q. f. d.

Proposition 5. - Soit $\left\{x_{n}\right\}$ une suite de Cauchy pour la structure $\mathfrak{U}_{\text {, }}$ admettant une valeur $d^{\prime}$ adhérence a pour la topologie $\mathfrak{f}$. Alors cette suite converge vers a pour la topologie définie par $\mathfrak{Q} \ell$ (et à fortiori pour la topologie $\mathfrak{f}$ ).

Donnons nous en effet $\varepsilon>0$, et soit $N$ assez grand pour que les inegalités $n>N$ et $p>N$ entraînent $e\left(x_{n}, x_{p}\right)<\varepsilon$; en choisissant une suite d'entiers $p_{k}$ telle que $x_{p_{k}}$ tende vers $a$, on a, par passage à la limite et application de la proposition précédente, l'inégalité $e\left(x_{n}, a\right)<\varepsilon$. Cette dernière inégalité etant vraie ponr $n>N$, on en déduit qne $x_{n}$ tend rers $a$ pour la structure $\mathscr{~}$.

ThÉonìm I. - Hour que la distribution $\Theta$ soit régulière, il faut et il suffit que $V$ soil complète pour la structure $2 \mathcal{L}$.

a) La condition est nécessaire.

Si $\Theta$ est régulière nous savons qu'il existe un voisinage $U$ de $e$ dans $G$ tel que, pour tout $a \in V, \pi_{G}$ réalise un diffémorphisme de la composante connexe de $(e, a)$ dans $\Sigma_{a} \cap \pi_{G}^{-1}(U)$ sur $U$; et il existe un nombre $\alpha>0$ tel que $U$ contienne la boule de $G$ définie par $d(e, g) \leq \alpha$.

Soit alors $\left\{x_{n}\right\}$ une suite de CADCHY pour la structure $\mathcal{Q} \mathcal{R}$, et soit $N$ assez grand pour que les inégalités $p>N$ et $n>N$ entrainent $e\left(x_{n}, x_{p}\right)<\alpha$. Fixons $x_{n}(n>N)$; à chaque point $x_{p}$ d'indice $p>N$ nous pouvons faire correspondre un are $\Gamma_{p}$ issu de $\left(e, x_{n}\right)$ et contenu dans $\Sigma_{x_{n}} \cap \pi_{G}{ }^{l}(U)$, tel que $\pi_{v}\left(\Gamma_{p}\right)$ joigne $x_{n}$ à $x_{p}$, ət que la longueur de $\pi_{G}\left(\Gamma_{p}\right)$ soit inférieure à $\alpha$. De la suite $\pi_{G}\left(\Gamma_{p}\right)$ nous pourons extraire une suite partielle convergeant vers un arc $\gamma$ issu de $e$, contenu dans $U$, et de longueur inférieure $a$; cet arc $\gamma$

$\left({ }^{6}\right)$ Voir [4], p. 48 (corollaire du th. IX). 
admet dans $\Sigma_{x_{n}}$ un relèvement $\Gamma$ d'origine $\left(e, x_{n}\right)$, et $\Gamma$ est limite d'une suite partielle extraite de $\Gamma_{p}$. Par projection sur $V$ on en deduit qu'il est possible d'extraire, de la suite $\left\{x_{p}\right\}$, une suite partielle convergeant dans la topologie $t$. Comme cette suite est de $\mathrm{C}_{\Delta}$ UCHY pour la structure $\mathcal{Q} \mathcal{L}$, il résulte de la proposition 5 , que la suite $\left\{x_{p}\right\}$ est convergente pour la structure $\mathcal{Q} \mathcal{L}$.

b) La condition est suffisante.

Supposons $A$ non régulière. Il existe alors un point $a$ de $V$ et un are $\gamma$ joignant $e$ à un point $g_{0}$ dans $G$, tels que $\varphi(g, a)$ soit défini sur l'arc semi ouvert [ego[ est ne soit pas prolongeable en $g_{0}$; et on sait ([4], Ch. III, th. VI, p. 67) que $\psi(g, a)$ tend vers le point a l'infini de $V$ quand $g$ tend vers $g_{0}$ sur $\gamma:$ si $\left\{g_{n}\right\}$ est une suite de points de $\gamma$ convergeant vers $g_{0}$, la suite $x_{n}=\varphi\left(g_{n}, a\right)$ est une suite de CAUCHY pour la structure $\mathcal{L}$, non convergente. C. q. f. d.

\section{§ 4. Une condition de régularité suffisante.}

Le théorème 1 nous a fourni un critére permettant (en principe) de reconnaïtre si la distribution $\Theta$ est régulière. Mais ce critère a l'inconvénient de faire intervenir le groupe local engendré par $\theta$, c'est à dire de supposer $q u^{\prime}$ 'on a intégré localement le système différentiel $(2,3)$. Nous allons maintenant établir d'autres critères qui n'utilisent pas le groupe local $\varphi$, mais seulement les données ( $G$ et $\Theta$ ). Dans chacun des cas nous commencerons pax établir une condition suffisante qui sera ensuite transformée de façon à fournir une condition nécessaire et suffisante. La première s'appuie sur le principe suivant.

Proposimion 6. - Soient $V$ et $\bar{V}$ deux variétés portant chacune un $G$-groupe de transformations infinitésimales et soient $\mathcal{Q} \mathcal{Q}, \overrightarrow{\mathcal{Q}}$ les structures uniformes respectivement associées à ces groupes. Pour que $V$ soit complète pour la structure $\mathcal{Q} \mathfrak{l}$ il suffit que $\bar{V}$ soit complète pour $\overline{\mathcal{W}}$ et qu'il existe une application $f$ de $I$ dans $V$ satisfaisant aux condition suivantes

a) $f$ est propre (7) pour les structures de variété $\mathfrak{f}$ et $\overline{\mathfrak{f}}$.

b) $f$ est uniformément continue pour les structures $\mathscr{\mathcal { R }}$ et $\overline{\mathcal{Q}}$.

Soit en effet $\left\{x_{n}\right\}(n \geq 1)$ une suite de CAdOHY pcur la structure $\mathscr{Q}$ sur $V$; d'après b) la suite $y_{n}=f\left(x_{n}\right)$ est une suite de CADCHY pour $\overline{\mathscr{Q}}$ sur $\bar{V}$; soit $y_{0}$ sa limite. L'ensemble $\bar{e}$ constitue par tous les $y_{1}(i \geq 0)$ est compact pour $\mathfrak{f}$, done $e=f^{-1}(\bar{e})$ est compact pour $\mathfrak{f}$; on en déduit que la suite $\left\{x_{n}\right\}$,

(7) Rappelons qu'une application est dite propre si l'image réciproque d'un compact quelconque est un compact. 
qui est contenue dans $e$, admet au moins une valeur d'adhérence pour la topologie $\mathfrak{f}$; et comme cette suite est de CAUCHY pour la structure $\mathcal{Q} \mathcal{R}$, elle est convergente (application de la proposition 5). C. q. f. d.

Nous sommes done ramenés à chercher un critère permettant d'affirmer qu'une application de $V$ dans $\bar{V}$ est uniformément continue pour les structures $\mathcal{Q} \mathfrak{l}$ et $\overline{\mathcal{Q}}$.

Proposition 7.- Soient $V$ et $\bar{V}$ deux variétés sur lesquelles les distribu. tions $\Theta$ et $\bar{\Theta}$ définissent respectivement un G-groupe de transformations infini. tésimales, et soient $\mathcal{Q}$ et $\overline{\mathcal{U}}$ les structures uniformes associées. Pour qu'une application $f$ de $V$ dans $\bar{V}$ soit uniformément continue pour les structures $\mathcal{Q}$, $\overrightarrow{\mathcal{Q}}$, il suffit qu'il existe une application continue et bornée ${ }^{\left({ }^{8}\right)} x \rightarrow A(x)$ de $V$ dans l' espace $\mathfrak{L}(\mathfrak{g}, \mathfrak{g})$ des transformations linéaires de $\mathfrak{g}$, satisfaisant $\grave{a}$

$$
\delta f_{x} \circ \Theta_{x}=\bar{\Theta}_{f(x)} \circ A(x) \text {. }
$$

Démonstration. - Convenons de dire, pour abréger, qu' un arc $C$ de $V$ et un are $\gamma$ de $G$ sont homologues s'il existe un are $\mathrm{I}$, tracé dans une feuille $\Sigma$ de $\Theta^{*}$, telle que $C=\pi_{V}(\Gamma)$ et $\gamma=\pi_{G}(\Gamma)$. Pour montrer que $f$ est uniformément continue pour les structures $\mathcal{U}$ et $\overline{\mathcal{U}}$, il nous suffit d'établir la propriété suivante :

"Si l'arc $O$ de $V$ a un homologue $\gamma$ de classe $C^{1}$ dans $G$, alors $f(C)$ a un homologae $\bar{\gamma}$ dans $G$, et le rapport (longueur $\bar{\gamma}) /($ longueur $\gamma$ ) est bornés.

Soit done $\gamma$ un are de $G$, d'origine $e$, image de [01] dans l'application de classe $C^{1} t \rightarrow g(t)$; ses homologues dans $V$ sont définis par des équations de la forme

$$
x=\varphi[g(t), a]=X(t),
$$

où a désigne un point quelconque de $V$.

A l'arc $C$ défini par $(4,2)$ correspond dans $\bar{V}$ l'arc $\bar{C}$ d'équation

$$
y=f[X(t)]=Y(t)
$$

et nous avons

$$
Y^{\prime}(t)=\delta f_{X(t)} \cdot X^{\prime}(t)=\left[\delta f_{X(t)} \circ \delta \psi_{g(t)}^{a}\right] \cdot g^{\prime}(t)
$$

soit encore, en utilisant les relations $(1,3)$ et l' hypothese $(4,1)$

$$
Y^{\prime}(t)=\left[\delta f_{X(t)} \circ \theta_{X(t)]} \cdot g^{\prime}(t)=\left\{\bar{\Theta}_{Y(t)} \circ A[X(t)]\right\} \cdot g^{\prime}(t) .\right.
$$

(8) La norme de $\mathfrak{L}(\mathfrak{g}, \mathfrak{g})$ se déduit de celle de $\mathfrak{g}$. 
Désignons par $L(t)$ le champ de vecteurs invaríant à droite sur $G$, tel que $L_{g(t)}=g(t)$; et soit $M(t)=A[X(t)] \cdot L(t)$ le champ de vecteurs invariant à droite qui s'en déduit par l'endomorphisme $\left.A\left[X_{(}\right)\right]$. Nous aurons

$$
Y^{\prime}(t)=\bar{\Theta}_{Y(t)}[M(t)] .
$$

L'application $A$ ayant été supposée continue, l'application de $\mathbb{R} \times G$ dans $g$ definie par $(t, h) \rightarrow M(t)_{h}$ est continue; d'autre part, pour $t$ fixé, elle définit un champ de vecteurs invariant à droite; elle est done de classe $C^{1}$ en $h$. De plus, les champ de vecteurs invariants à droite ayant une norme constante, nous avons

$$
\left\|M(t)_{h}\right\|=\|M(t)\| \leq \alpha\|L(t)\|=\alpha\left\|g^{\prime}(t)\right\|
$$

où

$$
\alpha=\sup _{x \in V}\|A(x)\|
$$

L'arc $\gamma$ étant supposé de classe $O^{2}$, cela prouve que $\left\|M(t)_{h}\right\|$ est borné. Done l'équation différentielle

$$
\frac{d h}{d t}=[M(t)]_{h}
$$

admet une solution unique définie sur $[0,1]$ et satisfaisant à $h(0)=e$.

Désignons par $\bar{\gamma}$ l'are de $G$ admettant pour représentation paramétrique la fonction $h(t)$ ainsi obtenue ; et soit $Z(t)=\bar{\varphi}[h(t,) \bar{a}]$ où l'on a posé $\bar{a}=f(a)$ et où $\bar{\varphi}$ désigne le groupe loeal engendré par $\bar{\theta}$. Nous aurons

$$
\frac{d Z}{d t}=\overline{\boldsymbol{\theta}}_{Z(t)}[M(t)] \quad \text { et } \quad Z(0)=\bar{a}
$$

ce qui pronve que $Z(t)$ satisfait à la même équation différentielle $(4,3)$ que la fonction $Y(t)=f[X(t)]$, avec la même valeur initiale $Z(0)=Y(0)=f(a)$; cette équation différentielle $(4,3)$ satisfaisant aux hypothéses de CAUcHYLIPSCHITZ, on en déduit l'identité $Z(t) \equiv Y(t)$.

Ainsi l'arc $\bar{C}$ admet l'arc $\vec{\gamma}$ pour homologue dans $G$; et nous arons l'inégalité

$$
\left\|\frac{d h}{d t}\right\| \leq \alpha\left\|\frac{d g}{d t}\right\|
$$

ce qui prouve que le rapport $\frac{\text { longueur } \bar{\gamma}}{\text { longueur } \gamma}$ est borné par $\alpha$. C. q. f. d. 
Remarque. - Définissons un système de coordonnées locales sur chacune des variétés $V$ et $\bar{V}$, et soit $\left\{x^{t}\right\}$ [resp. $\left\{y^{j}\right\}$ ce système. Désignons par $\left\{L_{z}\right\}$ une base de $\mathfrak{g}$, et par $\left\{\xi_{\alpha}^{i}(x)\right\}$ [resp. $\bar{\xi}_{\alpha}^{i}(y)$ ] les composantes de $\Theta_{x}\left(L_{x}\right)$ [resp. $\left.\overline{\boldsymbol{\theta}}_{\nu}\left(L_{\alpha}\right)\right]$. La condition $(4,1)$ équivaut aux relations $\left({ }^{9}\right)$

$$
\xi_{\alpha}^{i}(x) \frac{\partial f^{\sigma}}{\partial x^{i}}=A_{\alpha}^{\beta}(x) \bar{\xi}_{\beta}^{\sigma}[f(x)]
$$

les coefficients $A_{a}^{\beta}(x)$ étant des fonctions continues et bornées sur $V$.

\section{§ 5. Premier critère.}

Nous allons d'abord examiner deux cas particuliers importants des résultats précédents.

a) Cas oì $\bar{V}=G$.

Le groupe $G$ peut être considéré comme agissant sur lui-même à gauche et tenir le rôle de la variété $\bar{V}$. Si nous désignons par $\left\{\lambda_{\alpha}^{\beta}(g)\right\}$ les composantes de $\left(L_{\alpha}\right)_{\rho}$ dans un système de coordonnées locales $\left\{g^{\beta}\{\right.$ sur $G$, et par $\rho_{\alpha}^{\beta}(g)$ la matrice inverse de $\lambda_{\alpha}^{\beta}(g)$, les conditions $(4,4)$ s $^{\prime}$ écrivent ici

$$
\xi_{\alpha}^{i}(x) \frac{\partial f^{\sigma}}{\partial x^{i}}(x) \rho_{\sigma}^{\beta}[f(x)]=A_{\alpha}^{\beta}(x) .
$$

En rapprochant la proposition $(4,2)$ et le théorème I, nous obtenons, dans ce cas particulier, le résultat suivant.

Proposition 8. - Pour que le G-groupe infinitesimal $\Theta$ soit régulier sur la variété $V$, il suffit $q u$ 'il existe une application propre $f$ de classe $C^{1}$ de $V$ dans $G$ telle que les quantités

$$
A_{\alpha}^{\beta}(x)=\xi_{\alpha}^{i}(x) \frac{\partial f^{\sigma}}{\partial x^{i}}(x) \rho_{\sigma}^{\beta}[f(x)]
$$

soient bornées.

En effet, la continuité des $A_{\alpha}^{\beta}(x)$ résulte ici des hypothèses

Si $G$ est de dimension 1 , on retrouve, aveo des hypothèses un peu plus restrictives, le résultat rappele au début de cet article.

Il est à noter que les quantities $A_{\alpha}^{\beta}(x)$ definies par $(5,1)$ ne dépendent que de l'application $f$ et de la base $\left\{L_{\alpha}\right\}$ choisie: elles ne dépendent pas des systémes de coordonnées locales.

( 9 ) Nous adoptons iei la convention des indices muets. 
b) Cas oì $\bar{V}$ est un espace homogène $G / H$.

Soit $H$ un sous groupe fermé de $G$ et soit $G / H$ l'espace homogène constitué par les classes d'équivalence à gauche modulo $H$ : on peut considérer que $G$ agit à gau she sur $G^{\prime} H$, et par conséquent appliquer la proposition 7 en prenant $\bar{V}=G / H$. Cette remarque, va nous permettre d'obtenir une con. dition nécessaile et suffisante de régularité.

Définition 1. - Soit $\Theta$ un $G$-groupe de trasformations infinitésimales sur une variété $V$. Nous dirons que $\Theta$ satisfait à la condition $\mathcal{C}_{1}$ s'il existe un sous groupe fermé $H$ de $G$, une application propre $f$ de $V$ dans $G / H$, et une application continue et bornée $A$ de $V$ dans $\mathcal{L}(\mathfrak{g}, \mathfrak{g})$, satisfaisant à

$$
\delta f_{x} \circ \Theta_{x}=\bar{\theta}_{f(x)} \circ A(x)
$$

où $\bar{\Theta}$ désigne le $G$-groupe infinitésimal associé à l'action (par translations gauches) de $G$ sur $G / H$.

Définimion 2. - Une partie $M$ de $V$ sera dite G-invariante si on a défini sur $M$ une structure $\mathcal{S}$ de variété séparée de classe $C^{2}$ et un $G$-groupe infinitésimal $\theta^{M}$ tels que l'injection $i$ de $M$ dans $V$ soit de classe $C^{2}$ pour la structure $\mathcal{S}$, et que sa différentielle satisfasse à

$$
\delta i \circ \theta^{M}=\Theta
$$

Thto Rime II. - Pour que le G-groupe infinitésimal $\Theta$ engendre un $G$-groupe global sur la variété $V$, il faut et (si $G$ est simplement connexe) il suffit que $V$ soit la réunion d' une famille $\mathfrak{O}$ de parties $G$-invariantes satisfaisant à la condition $\mathfrak{C}_{1}$.

a) La condition est nécessaire.

Si $\theta$ engendre un $G$-groupe global, nous pouvons considerer $V$ comme réunion des orbites de ses points. Si $M$ est l'orbite de $a$, prenons pour $H$ le sous groupe fermé de $G$ laissant $a$ invariant, et à chaque $x \in M$ faisons correspondre la classe d'équivalence à gauche modulo $H$ constituée par les éléments $q \in G$ tels que $\varphi(g, a)=x$. Nous définissons ainsi une application biunivoque $f$ de $M$ sur $G / H$; l'image réciproque par $f$ de la structure de variété de $G / H$ est une structure de variété de classe $C^{\infty} \operatorname{sur} M$; et l'application $f$ satisfait évidemment à la condition $(5,2)$ quand on prend pour $A$ la matrice unité. D'autre part l'injection $i$ de $M$ dans $V$ vérifie la relation

$$
\varphi^{a}=i \circ f^{-1} \circ p
$$


où $p$ désigne l'application canonique de $G$ sur $G / H$. L'application $f^{-1} \circ p$ étant intérieure et de classe $C^{\infty}$, la relation $(5,4)$ détermine entièrement $i$, et montre que $i$ est de classe $C^{2}$ comme $\varphi^{a}$.

Enfin, nous pouvons considérer $G$ comme agissaut à ganche sur $G / H$, le groupe infinitésimal associè $\bar{\theta}$ étant défini par

$$
\bar{\Theta}(L)_{p(g)}=\delta p\left(L_{g}\right)
$$

Par transposition dans $M$, on en déduit l'existence d'un G-groupe globa de difféomorphismes de $M$, le groupe infinitésimal associé étant défini par l'homomorphisme $\theta^{M}=\delta\left(f^{-1}\right) \circ \dot{\theta}$.

Or en différentiant la relation $(5,4)$ on obtient

$$
\delta i \circ \delta\left(f^{-1}\right) \circ \delta p\left(L_{g}\right)=\delta \varphi_{a}\left(L_{g}\right)=: \theta(L)_{\varphi a(g)}
$$

où $L$ désigne un élément quelconque de $g$. Posant $y_{i}^{*}=f^{-1}[p(g)]$ et $x=i(y)=\varphi^{a}(g)$ on en déduit la relation

$$
\delta i_{\nu} \circ \Theta^{N}(L)_{\nu}=\Theta(L)_{x}
$$

qui peut s'écrire symboliquement

$$
\delta i 0 \cdot \theta^{M}=\theta
$$

C. q. f. d.

\section{b) La condition est suffisante.}

Si $G$ est simplement connexe, il résulte immédiatement du théorème I et des propositions 6 et 7 que $G$ agit globalement sur chaque variéte $M \in \mathscr{K}$. Autrement dit, quels que soient $M \in \mathscr{K}$ et $b \in M$, il existe une application $\varphi_{M}^{b}$ de $G$ dans $M$ satisfaisant à

$$
\delta \varphi_{M}^{b}\left(L_{g}\right)=\theta^{*}(L)_{\nu} \quad\left[\text { ò̀ } y=\varphi_{M}^{b}(g)\right]
$$

et à $\varphi_{M}^{b}(e)=b$.

Dono $\bar{\varphi}_{M}^{b}=i \circ \varphi_{M}^{b}$ est une application de $G$ dans $V$ satisfaisant à

$$
\delta \bar{\varphi}_{M}^{b}\left(L_{g}\right)=\delta i_{\nu} \circ \delta \varphi_{M}^{b}\left(L_{g}\right)=\Theta(L)_{x}
$$

si l'on a posé $x=i(y)=\overline{\varphi_{M}^{b}}(g)$.

Posons $a=i(b)=\bar{\varphi}_{M}^{b}(e)$. L'application $\varphi_{M}^{b}$ coincide avec $\varphi^{a}$ aux points où celle-ci est définie; donc s'il existe nne autre variété $M^{\prime} \in \mathfrak{K}$ et un point 
$b^{\prime} \in M^{\prime}$ tel que $i\left(b^{\prime}\right)=a$, on a $\bar{\varphi}_{M}^{b}(g)={\overline{\varphi_{M}}}_{b^{\prime}}^{b^{\prime}}(g)$ pour $g$ assez voisin de $e$. Si on pose $y^{\prime}=\varphi_{M^{\prime}}^{b^{\prime}}(g)$ et $x^{\prime}=i\left(y^{\prime}\right)$, les relations

$$
\bar{\varphi}_{M}^{b}(h g)=\bar{\varphi}_{M}^{y}(h) \quad \text { et } \quad \bar{\varphi}_{M^{\prime}}^{b^{\prime}}(h g)=\bar{\varphi}_{M^{\prime}}^{y^{\prime}}(h)
$$

montrent que l'ensemble des $g \in G$ tels que $\overline{\varphi_{M}^{b}}(g)=\bar{\varphi}_{M^{\prime}}^{b^{\prime}}(g)$ est ouvert. Comme cet ensemble est fermé par suite de la continuité des $\varphi$, il coìncide avec $G$ tout entier. La valeur de $\bar{\varphi}_{M}^{b}(g)$ ne dépend donc que des points $g$ et $a=i(b)$; si nous posons $\varphi_{M}^{b}(g)=\Phi(g, a)$, nous obtenons un prolongement de $\varphi(g, a)$ à tout $G$. Chaque point $a$ de $V$ appartenant a un ensemble $M$ an moins, on en déduit que le groupe infinitésimal $\Theta$ engendre bien un groupe global sur $U$.

\section{§ 6. Deuxième eritère.}

Comme nous l'avons déjà remarquè, le théorème II a été établi sous des hypothèses de régularité plus fortes que la proposition rappelée au début de cet article. Nous allons maintenant établir un critère constituant une généralisation plus immédiate de cette proposition. Pour l'énoncer, il nous faut d'abord généraliser la notion de dérivée de LrE.

\section{DáFInitions et nOtations.}

A chaque élément $L$ de $\mathfrak{g}$, nous faisons correspondre le sous groupe de $G$ à nn paramètre engendré par $L$, soit $\psi_{L}(t)=e^{L t}$, c'est à dire la solution de l'équation différentielle $\psi^{\prime}(t)=L_{\psi(t)}$ qui satisfait à $\psi(0)=e$

Soit alors $V$ une variété de classe $O_{1}^{1}$ portant un $G$-groupe local de transformations défini par l'application $\varphi(g, a)$ et soit $r(x)$ une fonction numérique (non nécessairement continue) définie sur $V:$ les nombres_-dérivés de Lie de $r(x)$ au point $a \in V$. relatifs à $L$, sont les valeurs d'adhérence, quand $t$ tend vers zéro, du rapport (défini pour $|t|$ assez petit):

$$
\frac{1}{t}\left[r \circ \varphi^{a} \circ \psi_{L}(t)-r(a)\right]
$$

[Si $r$ est de classe $O^{1}$ ce rapport a une limite $q$ ui est la dérivée de Lre de $r$ relative au champ $\Theta(L)]$.

Par la suite, la plus grande limite de la valeur absolue de ce rapport sera désignée par $X_{L}(r,(r)$; et nous dirons que la fonction $r$ a tous ses nom. bres dérivés de LIE bornés si, pour chaque $L \in \mathfrak{g}, X_{L}(r, x)$ est borné sur $V$ (cette borne pouvant dépendre de $L$ ). 
Enfin nous dirons que $r$ est $L$-Lipschitzienne de rapport $k$ si on a

$$
\left|r \circ \varphi^{a} \circ \psi_{L}(t)-r(a)\right| \leq k|t|
$$

pourra que $\varphi^{a}\left[\psi_{L}(t)\right]$ soit défini.

Proposition 9. - Pour que $r$ soit L-Lipschitzienne de rapport $k$, il faut et il suffit que $X_{L}(r, a)$ soit borné par $k$ quelque soit a.

La condition est évidemment nécessaire. Inversement supposons $X_{L}(r, a) \leq k$ quel que soit $a$, et posons $\rho=r \circ \varphi^{a} \circ \psi_{L}$. Nous aurons

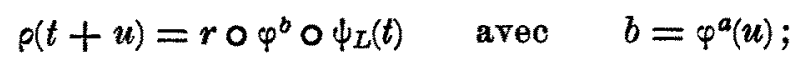

done

$$
\limsup _{t \rightarrow 0}\left|\frac{1}{t}[\rho(t+u)-\rho(u)]\right| \leq k
$$

ceci étant vrai quel que soit $t$ pourva que $\varphi^{a}\left[\psi_{L}(t)\right]$ soit défini, on en déduit l'inégalité

$$
|\rho(t)-\rho(0)| \leq k|t|
$$

c' est à dire $(6,1)$.

Proposition 10. - Pour que tous les nombres dérivés de Lie de $r$ soient bornés, il suffit que tous les nombres dérivés relatifs à une base $\left\{L_{x}\right\}$ de $\mathfrak{g}$ le soient; et les relations

$$
X_{L_{\alpha}} \leq k_{\alpha} \quad \text { et } \quad L=\underset{\alpha}{\Sigma} \lambda_{\alpha} L_{\alpha}
$$

entraînent (6.2) $X_{L} \leq \sum_{\alpha}\left|\lambda_{x}\right| k_{\alpha}$.

La proposition est évidente si $r$ est de classe $C^{1}\left[X_{L}(r, x)\right.$ étant alors la valeur absolue de la dèrivée de LiE de $r$ relative an champ $L$ ]. Pour l'établir dans le cas général, posons $R^{a}=r \circ \varphi^{a}$. Pour que $r$ soit $L$-Lipschitzienne de rapport $k$ sur $V$, il est nécessaire et suffisant que $R^{a}$ le soit sur $G$ pour tout $a \in V$ [le groupe $G$ étant consideré comme agissant a gauche sur lui-même]. Or si $R^{a}$ est $L_{\alpha}$-Lipschitzienne de rapport fini $k_{\alpha}$ pour chaque $\alpha$, la fonction $R^{a}$ est continue; et, dans un voisinage suffisamment petit de $e, R^{a}$ pent etre considéré comme limite d'ane suite $R_{n}^{a}$ de fonctions numérique de classe $C^{1}$, toutes $L_{\alpha}$-Lipschitziennes de rapport $k_{\alpha}$ pour chaque $\alpha$ : il suffit de considé rer une suite $S_{n}$ de voisinages compacts de $e$, convergeant vers $e$, et une 
suite $\omega_{n}$ de fonctions positives de classe $C^{1}$, la fonction $\omega_{n}$ ayant son support dans $S_{n}$ et satisfaisant à

$$
\int_{G} \omega_{n}(g) d \tau_{g}=1
$$

où $d \tau_{g}$ représente une mesure invariante à gauche sur $G$. Si $w$ est un voisinage symétrique de $e$ tel que $R^{d}$ soit défini dans $w \times w$, et si $n$ est assez grand pour que $S_{n}$ soit contenu dans $w$, alors l'intégrale

$$
R_{n}^{a}(g)=\int_{w \times w} \omega_{n}\left(h^{-1} g\right) R^{a}(h) d \tau_{h}
$$

est définie pour $g \in w$; et la suite $R_{n}^{a}$ ainsi obtenue satisfait aux conditions voulues. On a en effet, par une transformation simple

$$
R_{n}^{a_{n}}\left[\psi_{L}(t g]=\int_{w \times w} \omega_{n}\left(h^{-1} g\right) R^{a}\left[\psi_{L}(t) h\right] d \tau_{h}\right.
$$

d'où

$$
\left|R_{n}^{a}\left[\psi_{L_{\alpha}}(t) g\right]-R_{n}^{a}(g)\right| \leq k_{a}|t| \quad \nabla_{\alpha}
$$

Si pour ohaque $L \in \mathfrak{g}$ on pose

$$
Y_{L}\left(R_{n}^{a}, g\right)=\limsup _{t \rightarrow 0} \frac{1}{|t|}\left|R_{n}^{a}\left[\psi_{L}(t) g\right]-R_{n}^{a}(g)\right|
$$

on a done

$$
Y_{L_{\alpha}}\left(R_{n}^{a}, g\right) \leq k_{\alpha} \quad \nabla_{\alpha}
$$

$R_{a}^{n}$ étant de classe $C^{1}$, la relation $L=\sum_{\alpha} \lambda_{x} L_{x}$ entraine alors

$$
Y_{L}\left(R_{\mathfrak{n}}^{x}, g\right) \leq \Sigma_{\alpha}\left|\lambda_{\alpha}\right| k_{\alpha}
$$

et par application de la proposition 9 appliquée à $G$ lui-même, on en déduit l' inégalité

$$
\left|R_{n}^{\alpha}\left[\psi_{L}(t) g\right]-R_{n}^{a}(g)\right| \leq|t| \Sigma\left|\lambda_{\alpha}\right| k_{\alpha} .
$$


Si $n$ tend vers l'infini, on voit, à la limite, que $R^{a}$ est $L$-Lipschitzienne de rapport $\Sigma\left|\lambda_{\alpha}\right| k_{\alpha}$. C. q. f. d.

Proposition 11. - Pour que la fonctions $r$ ait tous ses nombres dérivés de Lie bornés, il faut et il suffit qu'il existe un nombre $k$ vérifiant l'inégalité

$$
|r(x)-r(a)| \leq k e(a, x)
$$

quels que soient $a, x \in V$.

RAPPEL. - Le groupe $G$ est supposé pourvu d'une métrique riemannienne invariante à droite, qui a servi à définir sur $V$ l'écart $e(x, y)$. A cette métríque est associee une norme sur $\mathfrak{g}$, et on a $\left\|L_{g}\right\|=\|L\|$ quels que soient $g \in G$ et $L \in \mathfrak{g}$.

a) Supposons l'inégalité $(6,3)$ vérifiée quels que soient $x$ et $a$. Si $\mid t$ est assez petit pour que $\psi_{L}(t)$ soit défini, on a:

$$
d\left[\psi_{L}(t), e\right] \leq\left|\int_{0}^{t}\left\|\psi_{L}^{\prime}(u)\right\| d u\right|=|t|\|L\|
$$

d'où

$$
\left|r \circ \varphi^{a} \circ \psi_{L}(t)-r(a)\right| \leq k|t||L| \mid
$$

ce qui entraine

$$
X_{L}(r, a) \leq k\|L\|
$$

b) Supposons que $r$ ait tous ses nombres dérivés de LIE bornés, et soit $\left\{L_{x}\right\}$ une base de $g$. La proposition 10 nous montre que $X_{L}$ admet une majoration de la forme $X_{L}(r, a) \leq k\|L\|$, où $k$ est indépendant de $L$. On à donc

$$
\left|r \circ \varphi^{a} \circ \psi_{L}(t)-r(a)\right| \leq k|t|\|L\|
$$

pourva que $\varphi^{a}\left[\psi_{L}(t)\right]$ soit défini.

Soit d'autre part $\varepsilon$ assez petit pour que la boule $d(e, g)<\varepsilon$ soit contenue dans un voisinage canonique de $e$. Si $e(a, x)<\varepsilon$, nous pouvons poser $x=p^{a}(g)$ avec $d(e, g)<\varepsilon$, et $g=\psi_{L}(1)$; de plus, si $\varepsilon \rightarrow 0, g$ tend vers $e$, et le rapport $d(e, g) /\|L\|$ tend vers l'unité. Pour $\varepsilon$ assez petit nous aurons donc $\|L\| \leq 2 d(e, g)$; et par application de $(6,4)$ on voit alors que l'inégalité entraine

$$
|r(x)-r(a)| \leq 2 k \varepsilon
$$


Soient alors deux points quelconques $a, x$ de $V$ tels que $e(a, x)$ soit fini ; et soit $n$ la partie entière de $e(a, x) \cdot \varepsilon$. De la façon dont $e(a, x)$ a eté défini, on déduit l'existence d'une chaine de points $x_{i}[i=0,1,2, \ldots, n+1]$ tels que $x_{0}=a, x_{n+1}=x$, et $e\left(x_{i}, x_{i+1}\right)<\varepsilon$ quel que soit $i$. On a done

$$
|r(x)-r(a)| \leq \sum_{i=0}^{n}\left|r\left(x_{i+1}\right)-r\left(x_{i}\right)\right| \leq 2(n+1) k \varepsilon \leq 4 k e(a, x)
$$

c'est à dire une inégalité de la forme $(6,3)$. C. q. f. d.

CoRollaire - Si la fonction numérique $r$ a tous ses nombrès dérivés de Lie bornés, alors $r$ est uniformément continue pour la structure $\longleftrightarrow$.

Ces préliminaires étant établés, nous obtenons sans peine le résultat général suivant, d'où découlera le denxième critère.

Proposinion 12. - Pour la variété $V$ soit complète pour la structure $\mathscr{W}$ définie par le G-groupe infinitésinnal $\Theta$, il suffit qu'il existe sur $V$ une fonction numérique $r(x)$, dont tous les nombres dérivés de Lie soient bornés, et telle que l'ensemble $E_{k}$ défini par $|r(x)| \leq k$ soit compact quel que soit $k$ pour la topologie $\mathrm{f}$.

Soit en effet $\left\{x_{n}\right\}$ une suite de CAUCHY sur $V$ pour la structure $\mathscr{Q}$; la fonction $r$ étant uniformément continue, la suite $\left\{r\left(x_{n}\right)\right\}$ est convergente, donc bornée; la suite $\left\{x_{n}\right\}$ est donc relativement compacte et admet une valeur d'adhérence $a$ pour la topologie $\mathfrak{f}$; il résulte de la proposition 5 que cette suite converge vers $a$ dans la structure $\mathcal{Q}$. C. $q$. f. d.

Pour énoncer commodément le deuxième critére, nous poserons la définition suivante

Défintrion. - Une variété $V$ portant un G-groupe infinitésimal $\Theta$ sera dite satisfaire à la condition $\mathcal{C}_{2} s^{\prime}$ il existe sur $V$ une fonction nunérique $r$ dont tous les nombres dérivés de Lie soient bornés et telle que $E_{h}=\{x \in V ; r(x) \leq k\}$ soit compact quel que soit $k$.

Nous pourrons alors énoncer.

Théoneme III. - Pour que le G-groupe infinitésimal $\Theta$ engendre un $G$-groupe global sur la variété $V$, il faut, et si $G$ est simplement connexe, il suffit, que $V$ soit la réunion de sous-variétés ouvertes $G$-invariantes $V_{\alpha}$ de même dimension satisfaisant à la condition $\mathfrak{C}_{2}$.

a) La condition est suffisante.

Si elle est réalisée, il résulte immédiatement de la proposition 12 et du théorème I que le $G$-groupe $\theta$ est régulier sur chaque $V_{z}$. Or si $a \in V_{\alpha} \cap V_{\beta}$, les feuilles $\Sigma_{a}^{x}$ et $\Sigma_{a}^{p}$ de $\Theta$ relatives à $V_{\alpha}$ et $V_{\beta}$ colncident: donc $\Sigma_{a}$ réalise, 
comme $\Sigma_{a}^{x}$ et $\Sigma_{b}^{\beta}$, un recouvrement de $G$. Si de plus $G$ est simplement connexe, la restriction de $\pi_{G}$ à $\Sigma_{a}$ est biunivoque, et $\Theta$ engendre un $G$-groupe global.

b) La condition est nécessaire.

Supposons que $\Theta$ détermine un $G$-groupe global sur $V$, et soit $\left|w_{z}\right|$ un recouvrement de $V$ par des cartes ouvertes relativement compactes. A chaque carte $w_{\alpha}$ faisons correspondre l'ensemble $V_{\alpha}=\varphi\left(G, w_{x}\right)$ : cet ensemble est ouvert, car e'est la réunion des ouverts $\varphi\left(g, w_{\alpha}\right)$ où $g$ parcourt $G$; et la restriction de $\varphi$ à $G \times w_{a}$ détermine sur $V_{a}$ un $G$-groupe global de diffé. omorphismes. Si $x \in V_{a}$ désignons par $r_{\alpha}(x)$ la borne inférieure des nombres $d(e, g)$ où $g \in G$ est assujetté à la condition $\varphi\left(g^{-1}, x\right) \in w_{x}$. La fonction $r_{\alpha}(x)$ ainsi définie sur $V_{\alpha}$ satisfait aux conditions voulues : en effet, d'abord, l'ensemble défini par $r_{\alpha}(x) \leq k$ est contenu dans le compact $\varphi\left(B_{k}, w_{\alpha}\right)$ où $B_{k}$ désigne la boule fermée définie par $d(e, g) \leq k$ dans $G$. D'autre part, si $y=\varphi(h, x)$ et si $\varphi\left(g^{-1}, x\right) \in w_{\alpha}$, alors $\varphi\left(g^{-1} h^{-1}, y\right) \in w_{\alpha}$. On en déduit l'inégalité

$$
r_{a}(y) \leq d(e, h g)=d\left(h^{-1}, g\right) \leq d\left(e, h^{-1}\right)+d(e, g)
$$

puis, en faisant varier successivement $g$ et $h$

$$
r_{\alpha}(y) \leq r_{\alpha}(x)+d\left(e, h^{-1}\right)
$$

et

$$
r_{a}(y) \leq r_{a}(x)+e(x, y)
$$

En échangeant les rôles de $x$ et $y$, on en déduit

$$
\left|r_{\alpha}(y)-r_{\alpha}(x)\right| \leq e^{\prime}(x, y),
$$

ce qui prouve d'après la proposition 11 , que $r_{z}$ a tons ses nombre dérirés de LIE bornés.

\section{ApPlication.}

Proposinion 13. - Soit $V$ une variété complète pour la métrique riemannienne $d s^{2}=g_{i j} d x^{i} d x^{j}$;

et soit $\Theta$ un G-groupe de transformations infinitésimales de $V$ tel que, pour chaque $L \in \mathfrak{g}$, les rapport des formes quadratique $\left({ }^{10}\right) X_{L} g_{i j} \alpha^{i} \alpha^{i} / g_{i j} \alpha^{i} \alpha^{i}$ soit borné. Alors $\Theta$ est régulier.

(10) $X_{L} g_{i j}$ désigne ici la dérivée de $L_{1 E}$ du tenseur $g_{i j}$ relative au champ $\xi_{L}(x)=\theta(L)_{x}$. 
Démonstration. - Désignons par $r(x)$ la distance gédésique de $x$ à un point fixe $x_{0}$ de $V$; on sait que l'ensemble $E_{k}=\{x ; r(x) \leq k\}$ est compact quel que soit $k$. Posons $\xi_{L}(x)=\Theta(L)_{x}$; on etablit comme dans [2] que les nombres dérivés de LIE de $r$ relatifs à $L$ satisfont à :

$$
X_{L}(r, x) \leq \sup \mid \xi_{L}(x) \cdot t_{k}(x, \mid
$$

où $\left\{t_{k}(x)\right\}$ désigne l'ensemble des vecteurs tangents en $x$ anx diverses géodésique joignant $x_{0}$ à $x$. Si l'on désigne d'autre part par $x(s)$ le point d'abscisse curviligne $s$ sur l'une de ces géodésiques, et par $\alpha^{i}(s)$ les composantes de $x^{\prime}(s)$, on a

$$
\frac{d}{d s}\left[\xi_{L}[x(s)] \cdot x^{\prime}(s)\right]=x^{\prime}(s) \cdot \frac{D \xi}{d s}=\xi_{i, j}^{L} \alpha^{i} \alpha^{j}=\frac{1}{2} X_{L} g_{i j} \alpha^{i} \alpha^{j}
$$

ou $\left\{\xi_{i}^{L}\right\}$ désigne les composantes covariantes de $\xi_{L}$. Et l'inégalité

$$
\left|X_{L} g_{i j} \alpha^{i} \alpha^{i} / g_{i j} \alpha^{i} \alpha^{i}\right| \leq A,
$$

que nous pouvons supposer vérifiée quels que soient les nombres $\alpha^{i}$, entrałne ici

$$
\left|\frac{d}{d s}\left\{\xi_{L}[(s)] \cdot x^{\prime}(s)\right\}\right| \leq \frac{A}{2}
$$

puisque $g_{i j} \alpha^{i}(s) \alpha^{j}(s)=1$.

Par intégration, on obtient l'inégalité

$$
\left|\xi[x(s)] \cdot x^{\prime}(s)\right| \leq \frac{A}{2} r(x)+\left|\xi\left(x_{0}\right)\right| .
$$

On a done

$$
\left|X_{L}(r, x)\right| \leq \frac{A}{2} r(x)+\left|\xi\left(x_{0}\right)\right|
$$

d' où, en posant

$$
p(x)=\log \left[r(x)+\frac{2}{A}\left|\xi\left(x_{0}\right)\right| \mid\right.
$$

l'inégalité

$$
\mid X_{L}(\rho, x) \leq \frac{A}{2}
$$


La fonction $\rho(x)$ a tous ses nombres dérivés de Lre bornés; et l'ensemble $E_{k}{ }^{\prime}=\{x ; \rho(x) \leq k\}$ est compact quel que soit $k:$ le résultat annoncé découle du thø̧orème III.

Remarque. - Les hypothèses faites sont réalisées en particulier si $\Theta$ est un groupe d'isométries (cas où $X_{L} g_{i j}=0$ ) ou si $\Theta$ est nn groupe de transformations conformes à coefficient borné [cas où $X_{L} g_{i j}=\lambda(L, x) g_{i j}$, avec $|\lambda|$ borné pour chaque $L \in \mathfrak{g}$ ].

\section{BIBLIOGRAPHIE}

[1] J. LeLong-FerRand, Sur les champs de vecteurs définissant un groupe d'homéomorphismes d' une variéte différentiable, * C. R. Acad. Sc. *, 245 (1957) 1491.1493.

[2] - - Sur groupes à un paramètre de transformations des variétès differentiables, $J$. Math. pures appl. *, 37 (1958), 269.278.

[3] - -, Condition pour qu'un groupe de transformations infinitesimales engendre un groupe global, * C. R. Acad. Se. *, 249 (1959), 1852.1854.

[4] R. S. Palais, A global formulation of the Lie theory of transformation groups, . Memoirs of the Amer. Math. Soc. s, n. 22 (1957). 